\title{
Dental Care for Patients With Childhood Cancers
}

\author{
Priyanshi Ritwik, BDS, MS \\ Department of Pediatric Dentistry, Louisiana State University Health Sciences Center School of Dentistry, New Orleans, LA
}

Background: Childhood cancer is the second most common cause of death in children in the United States. Treatments for childhood cancers usually incorporate chemotherapy, radiation therapy, immunotherapy, and/or surgery. This review of contemporary literature highlights the necessity for interprofessional care to achieve the best dental outcomes for this patient population during cancer treatment, as well as for children who are long-term cancer survivors.

Methods: A literature search at PubMed identified research and review articles about oral and dental complications in children diagnosed with cancer. Also included were evidence and current best practices from guidelines published by the American Academy of Pediatric Dentistry on dental management of pediatric patients receiving chemotherapy, hematopoietic cell transplantation, and/or radiation therapy. Finally, clinical recommendations from the European Society for Medical Oncology Guidelines Working Group on management of oral and gastrointestinal mucositis were included. The literature search was limited to articles published in the English language.

Results: Children with a new diagnosis of cancer should have dental care provided by a pediatric dentist to mitigate the oral complications from cancer treatment as well as to avoid dental infections during immunosuppressed states. An individualized oral care program can be developed for each patient to manage and treat acute and long-term oral complications related to cancer treatment.

Conclusion: Childhood cancer survivors present with unique challenges for dental management because of dental anomalies caused by cancer therapy. Interprofessional collaborative care between the pediatric dentist and the oncologist during and after cancer therapy will enable best outcomes for the pediatric patient. In addition, dental professionals must be prepared to meet the unique needs of long-term cancer survivors because of the increasing survival rates of childhood cancers.

Keywords: Childhood cancer survivors, dental health services, pathology-oral, periodontal diseases, pediatric dentistry

Address correspondence to Priyanshi Ritwik, BDS, MS, Department of Pediatric Dentistry, Louisiana State University Health Sciences Center School of Dentistry, 1100 Florida Ave., Box 139, New Orleans, LA 70119. Tel: (504) 352-8038. Email: pritwi@lsuhsc.edu

\section{INTRODUCTION}

Although childhood cancer accounts for $<1 \%$ of cancer diagnoses each year in the United States, it is the second leading cause of death in children. ${ }^{1,2}$ The American Cancer Society estimated that 10,590 children under the age of 15 in the United States were diagnosed with cancer in $2018 .{ }^{1}$ The diagnosis of a childhood malignancy leads to commencement of oncology treatment soon after. These children may receive chemotherapeutic agents, radiation therapy, immunotherapy, surgery, and/or hematopoietic cell transplantation (HCT) to treat the underlying malignancy. The long-term survival of children treated for malignancies is improving, with more than $80 \%$ of children surviving 5 years or more. ${ }^{1}$

Assessment of the oral cavity and management of dental complications is important for all children but particularly for children with cancer. Dental and oral care-related problems arise in these children at various phases of cancer treatment and after completion of cancer treatment. From a dental perspective, the pediatric patient undergoing oncology treatment may (1) have preexisting untreated dental caries, periodontal disease, and/or pathologic lesions of the oral hard and soft tissues; (2) have oral manifestations of the cancer; (3) develop oral complications as a result of cancer therapies; and (4) develop long-term dental and orofacial complications after completion of cancer therapies.

As a first step in developing this review of the topic, a literature search at PubMed identified research and review articles about oral and dental complications in children diagnosed with cancer. Also included were evidence and current best practices from guidelines published by the American Academy of Pediatric Dentistry on dental management of pediatric patients receiving chemotherapy, HCT, and/or radiation therapy. Finally, clinical recommendations from the European Society for Medical Oncology Guidelines Working Group on management of oral and gastrointestinal mucositis were included. The literature search was limited to articles published in the English language. 


\section{PRETREATMENT ORAL EXAMINATION}

Current guidelines recommend that children should have an oral examination prior to initiation of cancer therapy. ${ }^{3} \mathrm{~A}$ pretreatment examination facilitates establishment of a dentist-child relationship before the onset of cancer treatment-related oral complications. The examination involves a clinical and radiographic evaluation of the oral cavity, enabling the pediatric dentist to establish caries prevention strategies and to provide anticipatory guidance based on the child's medical diagnosis and upcoming treatments. ${ }^{3}$ The dentist should report the child's dental and oral health status to the oncology team. ${ }^{3}$ This communication should include the severity of dental caries, the invasiveness of proposed dental treatment, and the presence of pathologic lesions, if any. If dental caries, periodontal disease, or pathologic oral lesions ${ }^{4,5}$ are diagnosed prior to initiation of cancer therapies, the necessary dental treatment should be provided within the safety net of medical clearances and without causing unnecessary delay to cancer treatment. ${ }^{3}$

\section{PREVENTIVE STRATEGIES}

Dental caries is a preventable disease, ${ }^{6}$ and caries prevention is the most important aspect of oral care for children undergoing cancer therapy. ${ }^{7,8}$ The child and parents should be counseled on oral hygiene techniques. ${ }^{6,7,9,10}$ The teeth and tongue should be brushed at least twice each day with a soft-bristled nylon toothbrush. Fluoridated toothpaste (with the American Dental Association seal of approval) should be recommended. ${ }^{11}$ A smear or rice-size amount of fluoridated toothpaste should be used for children younger than 3 years of age, while a pea-size amount should be used for children 3-6 years of age (Figure 1). ${ }^{11}$

Fluoride is caries preventive. ${ }^{11}$ Over-the-counter fluoridated toothpaste and mouthrinses with a low concentration of fluoride $(900-1,100 \mathrm{ppm})$ can be used at home. ${ }^{11}$ Professional application of fluoride in the form of fluoride varnish, fluoride gel, or fluoride foam deliver much higher concentrations of fluoride $(12,300-22,600 \mathrm{ppm})$ and are administered in the

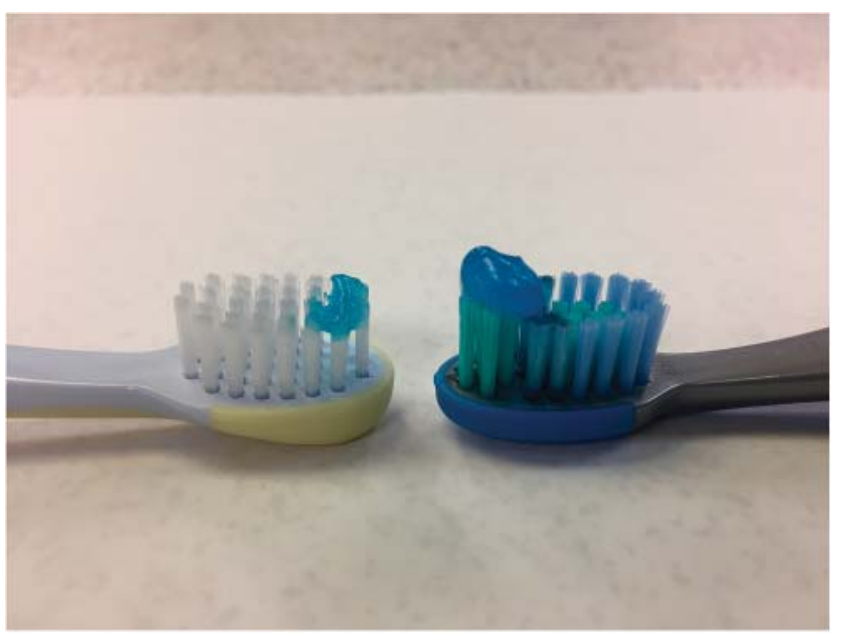

Figure 1. A smear of fluoridated toothpaste (left) is recommended for children younger than 3 years, whereas a peasize amount of toothpaste (right) is recommended for children 3-6 years. dental clinic. ${ }^{11}$ The frequency of professional fluoride application should be based on the patient's caries risk assessment. $^{12}$ Prescription high-strength fluoride toothpastes containing 5,000 ppm of fluoride should be prescribed to patients who can reliably expectorate toothpaste. ${ }^{12}$

Flossing is recommended for children who exhibit the necessary dexterity. ${ }^{3}$ Use of mouthrinses as an adjunct to brushing and flossing can be recommended if the child can swish and spit the mouthrinse.,12 Fluoridated mouthrinses can be recommended for children with clinical decay and/or demineralization of enamel. Children who have plaque-induced gingivitis or periodontal disease should be prescribed a chlorhexidine mouthrinse. ${ }^{3}$ An alcohol-free formulation of chlorhexidine mouth rinse may be better tolerated by children, especially during episodes of mucositis. ${ }^{3}$

Dietary counseling should include identification of noncariogenic foods that do not contain fermentable carbohydrates. $^{12}$ Consumption of sweetened beverages such as juices and sodas should be limited. ${ }^{12}$ Given the emotional circumstances of having a child with cancer, parents may be tempted to provide the child with comfort foods that are rich in sugar or carbohydrates. However, the parents and child need to be counseled on the importance of diet in preventing dental caries and minimizing complications during cancer treatment.

Children who receive radiation cancer therapy to the head and neck region may develop trismus. ${ }^{13-15}$ Physical therapy in the form of stretching exercises for masticatory muscles should be started prior to the initiation of radiation therapy. The patient should be encouraged to continue the exercises during and after radiation therapy. ${ }^{3}$

\section{DENTAL TREATMENT}

When dental caries is diagnosed in a child who is about to commence cancer treatment, the pediatric dentist and oncology team must establish clear communication regarding the ability of the patient to tolerate dental treatment. ${ }^{3,16}$ Some key medical considerations for the safe delivery of dental treatment (restorative treatment, periodontal therapy, and extractions) are absolute neutrophil count (ANC), platelet count, and coagulopathies secondary to cancer or treatment. ${ }^{3,16-18}$

When the ANC is $>2,000 / \mathrm{mm}^{3}$, antibiotic prophylaxis is not indicated. An ANC of $1,000-2,000 / \mathrm{mm}^{3}$ warrants a consultation with the pediatric oncologist to ascertain the need for antibiotic coverage. If the ANC is $<1,000 / \mathrm{mm}^{3}$, dental treatment should be deferred until the ANC is higher. For emergent dental treatment, the child may need prophylactic antibiotics and hospitalization., 38

Knowing the platelet count is important even if dental extractions are not planned because of the risk of bleeding and/or hematoma from intraoral anesthetic injections. Bleeding is not a concern when the platelet count is $>75,000 /$ $\mathrm{mm}^{3}$. If the child's platelet count is $<75,000 / \mathrm{mm}^{3}$, consultation with the pediatric oncologist is necessary to ascertain the need for platelet transfusions and arrange for hospitalization for prolonged monitoring. ${ }^{3,18}$

The pediatric dentist has to make decisions about the details of dental treatment, such as the choice of restorative materials and treatment techniques, knowing that the child is likely to be immunosuppressed in the near future. Ideally, all the dental treatment should be completed prior to 
commencement of cancer treatment. ${ }^{3,17,18}$ If this timing is not feasible because of urgency to begin therapy, the dentist is likely to prioritize the treatment of dental infections, extractions, periodontal therapy, and elimination of sources of intraoral tissue irritation.

Opinions differ among pediatric dentists regarding the use of therapeutic pulpotomy to treat vital primary teeth with reversible pulpitis., ${ }^{3,19}$ In the absence of evidence-based recommendations for vital pulp therapy in immunocompromised children, pediatric dentists tend to favor providing definitive treatment in the form of extraction of the primary tooth to avoid complications in the upcoming immunosuppressed state. ${ }^{18}$ If previously treated primary teeth are clinically and radiographically sound, extraction is not indicated. ${ }^{3,18}$ Root canal treatment should be performed for permanent teeth when indicated, provided they are restorable..,18 Ideally, root canal treatment for permanent teeth should be completed at least 1 week prior to initiation of cancer treatment to ensure healing of the periapical periodontal tissue. If this timing is not feasible, extraction should be considered.

Because of advances in adhesive dentistry, small carious lesions can be treated conservatively. Susceptible grooves and fissures should be sealed to prevent carious lesions on sound tooth surfaces. ${ }^{3,18}$ A method for arresting carious lesions is the application of silver diamine fluoride. Clinical success in arresting tooth decay in children has been reported with repeated applications of silver diamine fluoride. $^{20}$ However, application results in black discoloration of the tooth surface and should therefore be clearly discussed with the patient and the parents. ${ }^{20}$ Topical application of silver diamine fluoride allows the possibility of arresting decay with a noninvasive technique. ${ }^{20}$

Some children may have existing orthodontic appliances or space maintainers. Intraoral appliances should be removed if they have the potential to cause oral irritation, if the cancer therapy has the potential to cause moderate to severe mucositis, or if oral hygiene is poor. ${ }^{3,21}$ Removable appliances can be worn as long as the patient can tolerate them and is able to maintain good oral hygiene. ${ }^{3,21}$ Appliances with metal components should be removed from children who require multiple magnetic resonance images (MRIs) of the head and neck region (such as children with intracranial tumors) to prevent scatter and artifacts on the MRls. If the patient is undergoing orthodontic treatment at the time of cancer diagnosis, a prudent course is to expeditiously remove the orthodontic appliance and provide the patient with removable orthodontic retainers. ${ }^{21}$ Orthodontic treatment may be resumed after a 2-year event-free survival period. $^{21}$

Primary teeth in exfoliative stages should be allowed to naturally exfoliate. ${ }^{3,18}$ Infected teeth, nonrestorable teeth, root tips, and periodontally compromised teeth should be extracted 1-2 weeks prior to initiation of cancer therapy to allow time for healing. 3,18

\section{ORAL COMPLICATIONS RELATED TO CANCER TREATMENT}

Children usually receive chemotherapy in cycles or phases. ${ }^{3,18}$ Acute oral complications arise 5-7 days from the beginning of each cycle, corresponding to the drop in blood counts. Blood counts rise to normal levels after 21
Table. World Health Organization Oral Toxicity Scale ${ }^{28}$

\begin{tabular}{ll}
$\begin{array}{l}\text { Mucositis } \\
\text { Grade }\end{array}$ & \multicolumn{1}{c}{ Features } \\
\hline 1 & Soreness and/or erythema \\
2 & Erythema, ulcers; patient is able to swallow food \\
3 & $\begin{array}{l}\text { Ulcers, extensive erythema; patient is unable } \\
\text { to swallow food }\end{array}$ \\
4 & $\begin{array}{l}\text { Mucositis to the extent that alimentation } \\
\text { is not possible }\end{array}$ \\
\hline
\end{tabular}

days and remain so until the next cycle of chemotherapy begins. $^{3}$ Appropriate oral hygiene regimens, such as tooth brushing, should continue during episodes of thrombocytopenia and neutropenia. ${ }^{3,18}$

\section{Oral Mucositis}

Oral mucositis is likely to develop in $40 \%$ of children receiving standard-dose chemotherapy and in $80 \%$ of patients receiving radiation therapy for head and neck cancers. ${ }^{3,18,22-24}$ Oral mucositis occurs in $75 \%$ of patients undergoing bone marrow tranplantation. ${ }^{23-26}$ Oral mucositis is graded as mild, moderate, or severe based on the patient's symptoms and clinical presentation. Different rating scales can be used to assess and track cancer treatment-induced mucositis. ${ }^{27}$ The World Health Organization Oral Toxicity Scale is one such grading tool (Table). ${ }^{28}$ On this scale, grade 3 and grade 4 mucositis are considered to be severe. Oral mucositis interferes with patient functioning and tolerance for cancer therapy. ${ }^{29}$ Patients report it as the most debilitating side effect of cancer treatment. ${ }^{25}$

The mainstay of management of oral mucositis is palliative care. $^{3,25}$ Maintaining good oral hygiene is important to prevent as well as to reduce the severity of oral mucositis. ${ }^{30,31}$ Softening toothbrush bristles in warm water for a few minutes may be necessary to facilitate comfort during brushing. Patients who have severe mucositis and cannot tolerate a toothbrush can use foam brushes. ${ }^{3}$ Oral cryotherapy, recombinant human keratinocyte growth factor-1, low-level laser therapy, sodium bicarbonate rinses, and benzydamine mouthwash have evidence-based support for management of oral mucositis in patients with cancer. ${ }^{25}$ Analgesic medications can be used to treat pain associated with oral mucositis. ${ }^{3,25}$ Topical anesthetics obtund pain for a short duration but do not treat the mucositis. ${ }^{3,25}$ Further, because of systemic absorption from the oral mucosa, topical anesthetics can have cardiovascular and central nervous system effects and therefore should be used judiciously in young children, if at all.

\section{Oral Infections}

Children receiving cancer therapy develop opportunistic oral infections (fungal, bacterial and viral). ${ }^{30}$ The clinical manifestations of these infections may be atypical because of neutropenia. ${ }^{18}$ Oral candidiasis and herpetic infections are often seen in these children. ${ }^{18}$ Prophylactic nystatin is ineffective in preventing oral candidiasis. ${ }^{32}$ When oral candidiasis is diagnosed in children receiving cancer therapy, nystatin is the first line of medication to be tried, although 
it may not always resolve the infection. Systemic antifungal agents such as amphotericin B may be necessary. ${ }^{32}$ When nystatin is used, clinicians must keep in mind that the sugar content of the oral suspension is high and can increase caries susceptibility. ${ }^{33}$

\section{Neuropathic Pain}

Children who receive plant alkaloid chemotherapeutic agents such as vincristine and vinblastine may develop neuropathic pain that usually affects mandibular teeth. These children complain of deep pain in the jaw and teeth in the absence of an odontogenic source of pain. ${ }^{3,18,23}$ Such neuropathic pain is usually transient in children and diminishes or resolves after completion of chemotherapy. ${ }^{3}$ In the absence of a definitive cure for chemotherapy-induced neuropathic pain, palliative care may be provided with over-thecounter pain medications.

\section{Xerostomia}

Children who receive cancer chemotherapy and/or head and neck radiation therapy develop xerostomia during and beyond the treatment phase. ${ }^{3,16,18}$ Xerostomia increases caries risk and exacerbates mucositis., ${ }^{3,18}$ Use of sugarfree gum, sugar-free mints and lozenges, saliva substitutes, alcohol-free mouthrinses, and oral moisturizers helps alleviate xerostomia., ${ }^{3,18}$ Patients with xerostomia should also be encouraged to sip water frequently. ${ }^{3,16,18}$

\section{Lip Care}

Children receiving chemotherapy and/or radiation therapy often develop chapped lips and angular cheilitis. Lanolin-based creams and ointments are more effective in moisturizing the lips for these patients than petrolatumbased products. ${ }^{3}$

\section{ORAL CONCERNS RELATED TO HEMATOPOIETIC CELL TRANSPLANTATION}

Children being treated with HCT receive all the chemotherapy and/or total body irradiation just a few days before the transplant. $^{18}$ They have a prolonged immunosuppressed phase, ${ }^{18}$ and elective dental treatment cannot be performed during this immunosuppressed period. Immunologic recovery usually occurs at least 100 days after HCT; recovery time may be longer if graft-vs-host disease (GVHD) or other complications develop. Therefore, completing dental treatment prior to the initiation of HCT is critical. In these children receiving $\mathrm{HCT}$, mucositis develops 7-10 days after initiation of conditioning and lasts up to 2 weeks after the end of conditioning. The intensity and severity of oral complications begin to decline 3-4 weeks after completion of transplantation. This period corresponds to phase III (engraftment to hematopoietic recovery), and the primary oral concerns during this phase are acute GVHD, xerostomia, hemorrhage, neurotoxicity, temporomandibular dysfunction, and exophytic oral lesions (eg, granulomas, papillomas). Phase IV (immune reconstitution) begins approximately 100 days post-HCT. The oral concerns during this phase are related to the chronic effects of the conditioning regimen and include xerostomia, craniofacial growth abnormalities, viral infections, chronic GVHD, and predisposition to oral squamous cell carcinoma.

\section{UNIQUE ORAL CHALLENGES OF CANCER SURVIVORS}

Dental professionals must be prepared to meet the unique needs of long-term cancer survivors because of the increasing survival rates of those with childhood cancers. Children who receive cancer therapy develop dental and craniofacial problems that manifest later in life. ${ }^{5}$ Some specific oral and dental manifestations of childhood cancer treatment include higher risk for dental caries, xerostomia, tooth agenesis, microdontia, enamel hypoplasia, and blunting of roots. ${ }^{34-36}$ Figure 2 is a panoramic radiograph of a 15-year-old male who was diagnosed with high-risk acute lymphocytic leukemia at 2 years of age. He received chemotherapy, radiation therapy, and HCT. His radiograph shows microdontia of all his permanent teeth with blunting and tapering of roots. $\mathrm{He}$ also has agenesis of his permanent third molars.

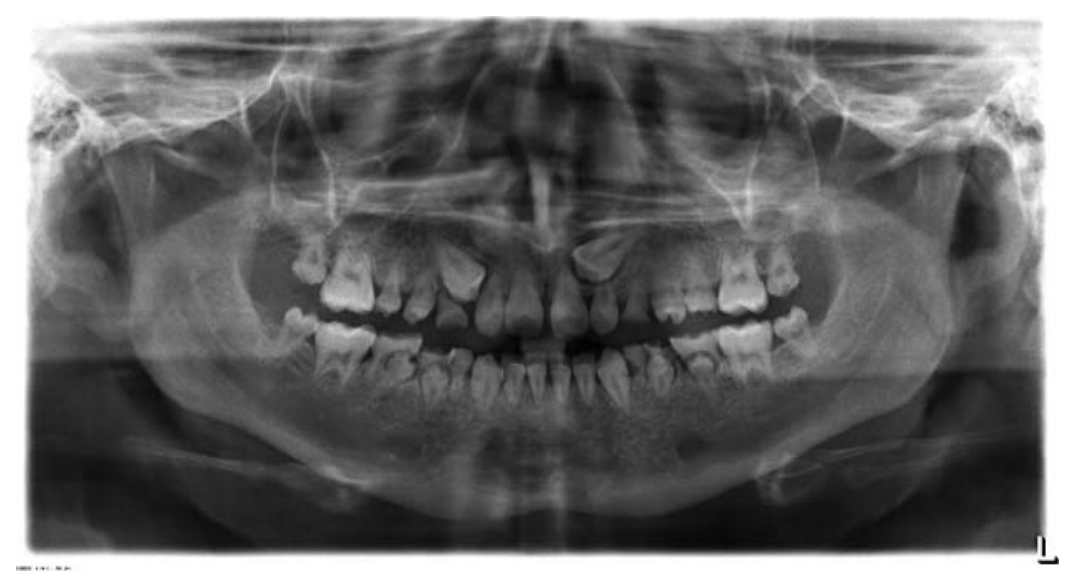

Figure 2. Panoramic radiograph of a 15-year-old male who was diagnosed with high-risk acute lymphoblastic leukemia at 2 years of age. He received chemotherapy, radiation therapy, and hematopoietic cell transplantation. Radiograph shows microdontia of all his permanent teeth, blunting and tapering of roots, and agenesis of permanent third molars. 


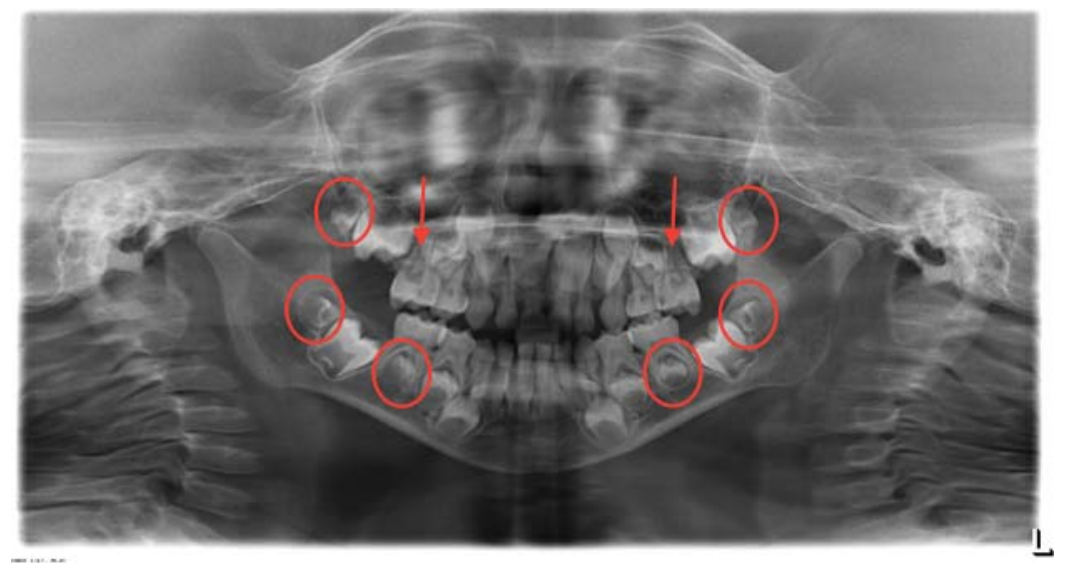

Figure 3. Panoramic radiograph of an 8-year-old male with DiamondBlackfan anemia who received chemotherapy and hematopoietic cell transplantation at age 3 years shows agenesis of his maxillary second premolars (red arrows), microdontia involving all 4 second permanent molars and mandibular second premolars (red ovals), and delayed development of the permanent dentition.

The permanent teeth affected and the severity of dental anomalies depend on the age of the child and stage of dental development at the time of cancer chemotherapy and/or radiation therapy, as well as the intensity of cancer therapy. ${ }^{37,38} \mathrm{~A}$ higher incidence of dental anomalies and developmental dental defects should be anticipated in children who receive cancer therapy before the age of 3 years. ${ }^{37}$ Figure 3 is a panoramic radiograph of an 8-year-old male with DiamondBlackfan anemia who received chemotherapy and HCT at the age of 3 years. His radiograph shows agenesis of his maxillary second premolars, microdontia involving all 4 second permanent molars and mandibular second premolars, and delayed development of the permanent dentition.

Childhood cancer survivors often experience xerostomia because of salivary gland hypofunction. ${ }^{34-36}$ Because of the increased caries risk, periodic dental examinations are important to assess and treat dental caries. Oral cancer screenings are critical for these patients. ${ }^{3}$ Suspicious soft tissue lesions should be referred to an oral and maxillofacial pathologist and surgeon for biopsy. ${ }^{3}$

Because of exposure to ionizing radiation, the developing craniofacial complex may exhibit malocclusion with a skeletal etiology. ${ }^{18}$ The orthodontic problems may be compounded by dental anomalies (tooth agenesis, microdontia). Often in teen years, these patients and their parents may desire orthodontic treatment. However, the caries risk because of xerostomia and enamel hypoplasia may prevent implementation of an ideal orthodontic treatment plan. If the teeth have blunted roots, orthodontic treatment is further limited as orthodontic movement of teeth can further reduce root length and lead to unfavorable crown-root ratios. The use of light orthodontic forces and compromised results should be discussed with the patient and parents. ${ }^{39}$ Figure 4 shows dental crowding, malocclusion, pulp chamber obliteration, and severe

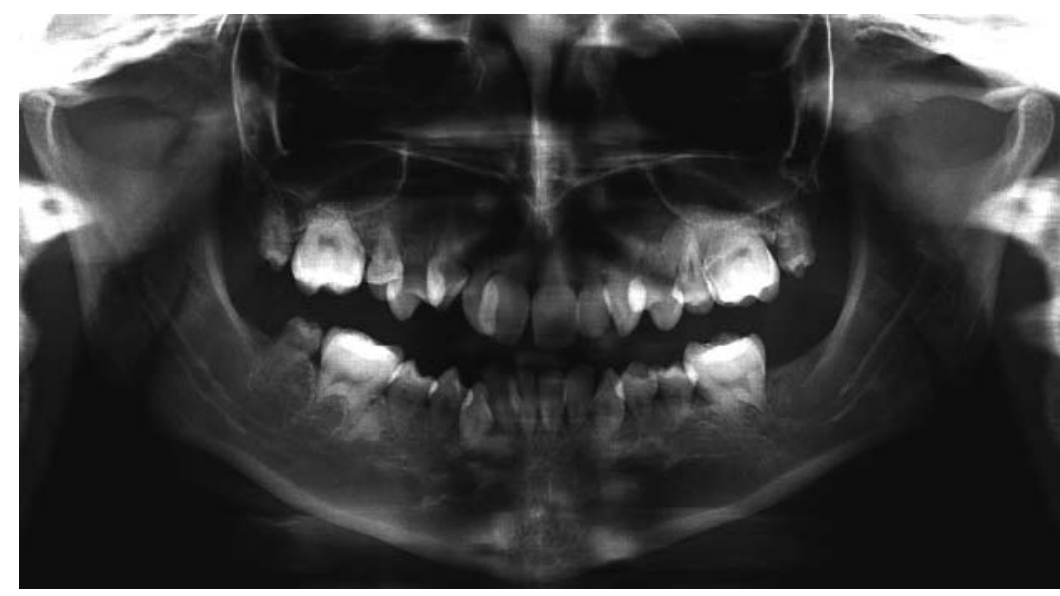

Figure 4. Panoramic radiograph of a 17-year-old shows dental crowding, malocclusion, pulp chamber obliteration, and severe shortening of the roots of all permanent teeth. The patient is a 15 -year neuroblastoma survivor. 
shortening of the roots of all permanent teeth in a 17-year-old who is a 15-year neuroblastoma survivor. Orthodontic treatment plans for childhood cancer survivors should take these limitations into consideration.

\section{CONCLUSION}

The pediatric dentist should be involved in the management of children with cancer from the time of cancer diagnosis so that an individualized preventive oral care program can be developed for each patient. Acute and long-term oral complications related to cancer treatment can be managed and treated in a patient-centered manner. The pediatric dentist needs the support of the oncology team to provide safe and effective dental treatment. Similarly, the oncology team needs the pediatric dentist to ensure that the child's oral cavity is disease-free to avoid complications during cancer therapy. Interprofessional collaborative care ensures patient comfort and best outcomes during and after cancer therapy.

\section{ACKNOWLEDGMENTS}

The authors have no financial or proprietary interest in the subject matter of this article.

\section{REFERENCES}

1. Cancer facts \& figures 2016. American Cancer Society. www .cancer.org/cancer/cancer-in-children/key-statistics.html?_ ga $=2.269021617 .1775200394 .1539201841-743091858$ .1539201841. Published 2018. Accessed October 10, 2018.

2. Childhood cancers. National Cancer Institute. www.cancer.gov /types/childhood-cancers. Published 2018. Accessed October 10, 2018.

3. Guideline on dental management of pediatric patients receiving chemotherapy, hematopoietic cell transplantation, and/or radiation therapy. Pediatr Dent. 2016 Oct;38(6):334-342.

4. Genc A, Atalay T, Gedikoglu G, Zulfikar B, Kullu S. Leukemic children: clinical and histopathological gingival lesions. J Clin Pediatr Dent. 1998 Spring;22(3):253-256.

5. Valéra MC, Noirrit-Esclassan E, Pasquet M, Vaysse F. Oral complications and dental care in children with acute lymphoblastic leukaemia. J Oral Pathol Med. 2015 Aug;44 (7):483-489. doi: 10.1111/jop.12266.

6. Policy on early childhood caries (ECC): unique challenges and treatment options. Pediatr Dent. 2017 Sept;39(6):62-63.

7. Hartnett E, Krainovich-Miller B. Preventive dental care: an educational program to integrate oral care into pediatric oncology. Clin J Oncol Nurs. 2017 Oct 1;21(5):611-616. doi: 10.1188/17.CJON.611-616.

8. Cubukçu CE, Güneş AM. Caries experience of leukemic children during intensive course of chemotherapy. J Clin Pediatr Dent. 2008 Winter;32(2):155-158.

9. Levin L, Bilder L, Borisov O. Improving oral hygiene skills among children undergoing treatment at the haematooncology department - an interventional programme. Int Dent J. 2015 Aug;65(4):211-215. doi: 10.1111/idj.12171.

10. Haytac MC, Dogan MC, Antmen B. The results of a preventive dental program for pediatric patients with hematologic malignancies. Oral Health Prev Dent. 2004;2(1):59-65.

11. Guideline on fluoride therapy. Pediatr Dent. 2016 Oct;38(6):181184.

12. Guideline on caries-risk assessment and management for infants, children, and adolescents. Pediatr Dent. 2016 Oct;38 (6):142-149.
13. Katz J, Peretz B. Trismus in a 6 year old child: a manifestation of leukemia? J Clin Pediatr Dent. 2002 Summer;26(4):337-339.

14. Wang CJ, Huang EY, Hsu HC, Chen HC, Fang FM, Hsiung CY. The degree and time-course assessment of radiation-induced trismus occurring after radiotherapy for nasopharyngeal cancer. Laryngoscope. 2005 Aug;115(8):1458-1460. doi: 10.1097/01.mlg.0000171019.80351.46.

15. Rapidis AD, Dijkstra PU, Roodenburg JL, et al. Trismus in patients with head and neck cancer: etiopathogenesis, diagnosis and management. Clin Otolaryngol. 2015 Dec;40 (6):516-526. doi: 10.1111/coa.12488.

16. Fleming P. Dental management of the pediatric oncology patient. Curr Opin Dent. 1991 Oct;1(5):577-582.

17. Farsi DJ. Children undergoing chemotherapy: is it too late for dental rehabilitation? J Clin Pediatr Dent. 2016;40(6):503-505. doi: 10.17796/1053-4628-40.6.503.

18. Hong $\mathrm{CH}$, daFonseca M. Considerations in the pediatric population with cancer. Dent Clin North Am. 2008 Jan;52 (1):155-181, ix. doi: 10.1016/j.cden.2007.10.001.

19. Halperson E, Moss D, Tickotsky N, Weintraub M, Moskovitz M. Dental pulp therapy for primary teeth in children undergoing cancer therapy. Pediatr Blood Cancer. 2014 Dec;61(12):22972301. doi: 10.1002/pbc.25227.

20. Chibinski AC, Wambier LM, Feltrin J, Loguercio AD, Wambier $D S$, Reis A. Silver diamine fluoride has efficacy in controlling caries progression in primary teeth: a systematic review and meta-analysis. Caries Res. 2017;51(5):527-541. doi: 10.1159/ 000478668.

21. Sheller B, Williams B. Orthodontic management of patients with hematologic malignancies. Am J Orthod Dentofacial Orthop. 1996 Jun;109(6):575-580.

22. Lowe O. Oral concerns for the pediatric cancer patient. $J$ Pedod. 1986 Fall;11(1):35-46.

23. Ponce-Torres E, Ruíz-Rodríguez Mdel S, Alejo-González F, Hernández-Sierra JF, Pozos-Guillén Ade J. Oral manifestations in pediatric patients receiving chemotherapy for acute lymphoblastic leukemia. J Clin Pediatr Dent. 2010 Spring;34 (3):275-279.

24. Curra M, Soares Junior LAV, Martins MD, Santos PSDS. Chemotherapy protocols and incidence of oral mucositis. An integrative review. Einstein (Sao Paulo). 2018;16(1):eRW4007. doi: 10.1590/s1679-45082018rw4007.

25. Peterson DE, Bensadoun RJ, Roila F; ESMO Guidelines Working Group. Management of oral and gastrointestinal mucositis: ESMO clinical practice guidelines. Ann Oncol. 2011 Sep;22 Suppl 6:vi78-84. doi: 10.1093/annonc/mdr391.

26. Chaudhry HM, Bruce AJ, Wolf RC, et al. The incidence and severity of oral mucositis among allogeneic hematopoietic stem cell transplantation patients: a systematic review. Biol Blood Marrow Transplant. 2016 Apr;22(4):605-616. doi: 10.1016/ j.bbmt.2015.09.014.

27. Jaroneski LA. The importance of assessment rating scales for chemotherapy-induced oral mucositis. Oncol Nurs Forum. 2006 Nov 27;33(6):1085-90; quiz 1091-1093. doi: 10.1188/06. ONF.1085-1093.

28. World Health Organization handbook for reporting results of cancer treatment. World Health Organization. Geneva, Switzerland; 1979. apps.who.int/iris/bitstream/handle/10665 /37200/WHO_OFFSET_48.pdf?sequence=1\&isAllowed=y. Accessed October 12, 2018.

29. Staudenmaier T, Cenzer I, Crispin A, Ostermann H, Berger K. Burden of oral mucositis in stem cell transplant patients-the patients' perspective. Support Care Cancer. 2018 May;26 (5):1577-1584. doi: 10.1007/s00520-017-4000-5. 
30. Glenny AM, Gibson F, Auld E, et al; Children's Cancer and Leukaemia Group (CCLG)/Paediatric Oncology Nurses Forum's (CCLG-PONF) Mouth Care Group. The development of evidence-based guidelines on mouth care for children, teenagers and young adults treated for cancer. Eur J Cancer. 2010 May;46(8):1399-1412. doi: 10.1016/j.ejca.2010.01.023.

31. Velten DB, Zandonade E, Monteiro de Barros Miotto MH. Prevalence of oral manifestations in children and adolescents with cancer submitted to chemotherapy. BMC Oral Health. 2017 Jan 20;17(1):49. doi: 10.1186/s12903-016-0331-8.

32. Morgan JE, Hassan H, Cockle JV, Lethaby C, James B, Phillips RS. Critical review of current clinical practice guidelines for antifungal therapy in paediatric haematology and oncology. Support Care Cancer. 2017 Jan;25(1):221-228. doi: 10.1007/ s00520-016-3412-y.

33. Clark SA, Vinson LA, Eckert G, Gregory RL. Effect of commonly prescribed liquid medications on streptococcus mutans biofilm. An in vitro study. J Clin Pediatr Dent. 2017;41(2):141146. doi: 10.17796/1053-4628-41.2.141.

34. Lauritano D, Petruzzi M. Decayed, missing and filled teeth index and dental anomalies in long-term survivors leukaemic children: a prospective controlled study. Med Oral Patol Oral Cir Bucal. 2012 Nov 1;17(6):e977-e980.
35. Avşar A, Elli M, Darka O, Pinarli G. Long-term effects of chemotherapy on caries formation, dental development, and salivary factors in childhood cancer survivors. Oral Surg Oral Med Oral Pathol Oral Radiol Endod. 2007 Dec;104(6):781-789. doi: 10.1016/j.tripleo.2007.02.029.

36. Nemeth O, Hermann P, Kivovics P, Garami M. Long-term effects of chemotherapy on dental status of children cancer survivors. Pediatr Hematol Oncol. 2013 Apr;30(3):208-215. doi: 10.3109/ 08880018.2013.763391.

37. Bagattoni S, D'Alessandro G, Prete A, Piana G, Pession A. Oral health and dental late adverse effects in children in remission from malignant disease. A pilot case-control study in Italian children. Eur J Paediatr Dent. 2014 Mar;15(1):45-50.

38. Wilberg P, Kanellopoulos A, Ruud E, Hjermstad MJ, Fosså SD, Herlofson BB. Dental abnormalities after chemotherapy in long-term survivors of childhood acute lymphoblastic leukemia 7-40 years after diagnosis. Support Care Cancer. 2016 Apr;24 (4):1497-1506. doi: 10.1007/s00520-015-2940-1.

39. Dahllöf $G$, Jönsson A, Ulmner M, Huggare J. Orthodontic treatment in long-term survivors after pediatric bone marrow transplantation. Am J Orthod Dentofacial Orthop. 2001 Nov;120 (5):459-465. doi: 10.1067/mod.2001.118102.

This article meets the Accreditation Council for Graduate Medical Education and the American Board of Medical Specialties Maintenance of Certification competencies for Patient Care, Medical Knowledge, and Systems-Based Practice. 